\title{
POWER FLOW ANALYSIS OF DOUBLY FED INDUCTION GENERATOR BASED 220/132KV POWER GRID
}

\author{
Arsalan Muhammad Soomar ${ }^{1}$, Abdul Hakeem Shaikh' ${ }^{1}$, Muhammad Hanif Lashari ${ }^{2}$ \\ ${ }^{1}$ Department of Electrical Engineering, Mehran University of Engineering and Technology, \\ Jamshoro, Pakistan \\ ${ }^{2}$ Department of Electrical Engineering, Quaid-e-Awam University of Engineering, \\ Science and Technology, Pakistan
}

\begin{abstract}
This paper proposes the analysis and review of different factors of wind farm connected grid station. the ideal situation is rarely mate by wind farm due to this similar condition for difference reasons. In this study the model is of wind farm connected grid is developed with maximum capacity. A data of for months in real time was collected, simulated results were analyzed and the same was reviewed carefully by increasing the capacity from $750 \mathrm{MW}$ to $1250 \mathrm{MW}$. The validity of results was found satisfactory by comparing the simulated results with the real data.
\end{abstract}

Key words: Power Flow analysis, Electrical Transient Analysis Program, Single line diagram, Wind Turbine Generator, Doubly-Fed induction generator

Cite this Article: Arsalan Muhammad Soomar, Abdul Hakeem Shaikh and Muhammad Hanif Lashari. Power Flow Analysis of Doubly Fed Induction Generator Based 220/132KV Power Grid. International Journal of Electrical Engineering and Technology (IJEET). 12(9), 2021, pp. 107-120.

https://iaeme.com/Home/issue/IJEET?Volume $=12 \&$ Issue $=9$

\section{INTRODUCTION}

In our power system renewable energy sources like solar, wind are increasing rapidly as electricity generation source, with inclusion of these sources in existing system the load flow (also known as power flow analysis) which is backbone and has great importance in the systems planning and operation as we know the need for electric power is exceeding and expanding day by day, load flow studies can play indispensable parts. Load flow analysis is more accurate which takes less time to calculate with highly reliable results by user friendly software ETAP Electrical Transient Analyzer Program to carry out the load flow analysis of the grid. This Jhimpir grid is located in the Sindh, interconnected to NTDC (National transmission and dispatch company).The major cause of all the leading disturbances caused in the power system is under voltages, reactive power is never be conveyed identical faraway, exclusively beneath 
substantial consignment situations so it obligation be In our power system load flow (also known as power flow analysis) which is backbone and have great importance in the systems planning and operation as we know the need for electric power is exceeding and expanding day by day, load flow studies can play indispensable parts. Load flow is considered as obligatory for system planning, operation, economical operations, development with exchanging of power among utility sections. Load flow investigation is further used in stability under transients, optimal flow of power and continuity or exigency studies Therefore, this increasing demand not only to overcome by building more generating stations but also to redesign and restructure the current power grids with advance techniques, furthermore the data of analysis of load flow which is useful to discovery out the degree, and angle in phase between voltages at every bus with the reactive and real power graceful and consumptions in separately transmission lines through the implementation of the software electrical transient and analysis program (ETAP) these all things are much more easier to find with accuracy more than human calculations by hand.

(Albadi, M., 2019) Analyzed the power flow by considering the simple power system and made the equation. Since these equations are nonlinear, that's why he used iterative techniques which used to solve these equations like Newton Raphson, gauss-seidel and the fast-decoupled methods, when he come through the solutions and he concluded in the results that he found fast decoupled method is faster and simplified form of newton Raphson method which is much reliable and faster method to solve the equations of power flow.

The power flow analysis of a $138 / 69 \mathrm{kV}$ grid station and examined different operating setups using ETAP and result obtained from the load flow analysis to improve the system performance by different approaches of power factor rectification with fixed and switched techniques which originates with plentiful technical and financial profits [1]. These techniques aids contain improvement in voltage stability and loss in power, with minimum equipment's loading, and reorganizing costly network advancements.

By constructing the one line diagram in software named Electrical transient analysis program, and with various cases he did the analysis of substation such as by doing loss of generators, a transmission line, a transformer and a load, and to determine the load analysis at various cases and self-made conditions and he also regulate the optimal size and area in which capacitor bank is to overcome the problem of under voltage [2].

In [3], ETAP software is used to support his research for the analysis of $220 / 132 \mathrm{kV}$ substation in which he carried out load flow analysis and found the problem of under voltages at the substation buses, and he found this software useful and excellent tool while doing the study, and system planning also to determine the optimum size and location of capacitor to triumph the underneath voltage problem and this software was originated this to be useful while connecting or disconnecting the loads to identify the system voltages under various cases to find out the system equipment's ratings.

In [4] author did the analysis of CIGRE Micro-grid Model which he simulated in ETAP software to analyze the load flow to determine its voltage Stability, the power flow both real and reactive among all the buses. The results stated that the power flows between the buses and loads then he also explained the system voltage at buses which he concludes that these results will be helpful during system physical implementation and planning. Further research work incorporates the load transients and transient's stability study.

In [5], the author did the Power flow analysis of power station 220/132 kV in ETAP. To carry out the study for future planning and expansion of the substation in addition to determine the best process for the present system which exist, after analysis he found the problem of under voltages, then he provide the solution of under voltage by adding a capacitor to overcome this issue to maintain the voltage levels under safe operating values of equipment. 
In [6], the author carried out power flow analysis for reasonable process of the power system. In this analysis he adopted the results by using simulation software Power World. In this software he also analyzes the effect of adding and removing of transmission line from the power system component, simulator results gives information for what amount of power the system works with maximum efficiency and he also found the software is user friendly which provides visual results.

In [7], the analysis of the $132 \mathrm{kV}$ grid voltages of two month then he observed the problem of under voltages by using the software ETAP, then he also provide the solution of that problem of under voltages by inserting the capacitor bank of 8MVAR in shunt with the feeders, then he compare the result and analyze the problem of under voltages and losses is reduced and voltages are not in critical situation.[8] Load flow analysis on 220/132kV transmission system of Maharashtra state ETCL, one-line diagram in MATLAB, then he recognized the overload bus and he anticipated appropriate procedures for decreasing the overload and advised. The advancement in the substation change in tapping of transformers, capacitive banks for power compensation, shifting techniques of capacitors, and substituting single lines circuits by double lines circuits were initiated to be operative in improving the issue of under voltages. In [9], the researcher did the Load flow analysis and verified the data of 220kV MSETCL substation by equating the results with three methods that is by hand calculations, the data collected by ABT metering installed at substation and ETAP, then results were found to be technically correct and time required is very less. The difference in the results is less than $1 \%$ for all bus voltages and $2 \%$ for all power flows, and he found the ETAP software was useful for this operation.

\section{POWER FLOW ANALYSIS}

The Immensity and phase angle of voltage, real and reactive power are the foremost extents that are accompanying with the bus in power system. Based on four extents, buses are confidential hooked on three classes two of which are quantified and the enduring two cannister be originate out by the resolution of the comparison.

\subsection{Load Bus}

Active and reactive power of load bus can be quantified nevertheless phase angle and greatness of the bus voltage is determined. The load bus that can be allowed to vary the voltage buy permissible value such as $5 \%$ would only to specify the active and reactive although the voltage phase angle is not that abundant significant.

\subsection{Generator Bus}

The rated real power and rated magnitude of voltage is quantified for bus. The outstanding two measures that are voltage phase angle and the reactive power cohort of the bus is strongminded. Generator bus is considered at generation stations or wherever power generation devices are installed.

\subsection{Swing Bus}

In this bus greatness and phase angle of the voltage is identified whereas the real power and reactive power is strongminded by the load flow resolution. This is the category of the generator bus that underwrites to the transmission losses to make additional real and reactive power supply. For this bus is acknowledged as swing bus. 
Table 1 Summary of three types of buses

\begin{tabular}{|l|l|l|}
\hline \multicolumn{1}{|c|}{$\begin{array}{c}\text { Bus } \\
\text { Cataloging }\end{array}$} & \multicolumn{1}{c|}{$\begin{array}{c}\text { Specified } \\
\text { Constraints }\end{array}$} & \multicolumn{1}{c|}{$\begin{array}{c}\text { Obtained } \\
\text { Constraints }\end{array}$} \\
\hline Generator Bus & $\begin{array}{l}\text { Voltage magnitude, } \\
\text { Active power }\end{array}$ & $\begin{array}{l}\text { Voltage angle, } \\
\text { Reactive Power }\end{array}$ \\
\hline Swing Bus & $\begin{array}{l}\text { Voltage magnitude, } \\
\text { Voltage Angle }\end{array}$ & $\begin{array}{l}\text { Active Power, } \\
\text { Reactive Power }\end{array}$ \\
\hline Load Bus & $\begin{array}{l}\text { Active Power, } \\
\text { Reactive Power }\end{array}$ & $\begin{array}{l}\text { Voltage magnitude, } \\
\text { Voltage angle }\end{array}$ \\
\hline
\end{tabular}

\section{RESEREACH METHODOLOGY}

ETAP is the electrical instrument its abbreviation electrical transient analyzer program, its prototypical ambitious construction affords the quicker and factual time data to its users, and it also offers a complete explanation to all the electrical complications such as power flow ,arc flash and short circuit scrutiny, transient stability relay synchronization, cable assortment to estimation the antedating manners, cautionary accomplishment and situational flair [10].Solitary of its untouchable assemblies is that it distributes the atmosphere for the unceasing functionality from deceitful to dispensation. It affords tailored amplification for any encompassing of a unimportant or bulky power arrangement. At this point we are absorbed on an extra high tension (EHT) line involvement valuation of $132 \mathrm{kV}$.

Simulating model of 220/132kV grid under study is developed in ETAP as shown in Fig.1. The model is made to simulate in order to determine the performance of grid Table I: Summary of Data collected from the grid for generating plants. The actual rating of the grid components such as transformers summary of data in Table V, circuit breakers, current transformers, potential transformers, isolators and various buses data summary at Table III, IV the data are taken from grid and modified accordingly in the ETAP.

Table 2 Summary of WTG Capacity and Ratings

\begin{tabular}{|l|c|c|c|}
\hline Generating Plants & $\begin{array}{c}\text { Control } \\
\text { Breaker }\end{array}$ & Capacity & $\begin{array}{c}\text { Voltage } \\
\text { Rating }\end{array}$ \\
\hline ARTISTIC & (E17Q1) & $50 \mathrm{MW}$ & $11 / 132 \mathrm{kV}$ \\
\hline MASTER & (E13Q1) & $49.5 \mathrm{MW}$ & $11 / 132 \mathrm{kV}$ \\
\hline Metro & (E5Q1) & $60 \mathrm{MW}$ & $11 / 132 \mathrm{kV}$ \\
\hline Sachal & (E7Q1) & $49.5 \mathrm{MW}$ & $11 / 132 \mathrm{kV}$ \\
\hline TBA & (E4Q1) & $50 \mathrm{MW}$ & $11 / 132 \mathrm{kV}$ \\
\hline TBC & (E3Q1) & $50 \mathrm{MW}$ & $11 / 132 \mathrm{kV}$ \\
\hline TGS & (E9Q1) & $50 \mathrm{MW}$ & $11 / 132 \mathrm{kV}$ \\
\hline TGT & (E8Q1) & $50 \mathrm{MW}$ & $11 / 132 \mathrm{kV}$ \\
\hline UEP & (E18Q1) & $99 \mathrm{MW}$ & $11 / 132 \mathrm{kV}$ \\
\hline ZEPHYR & (E14Q1) & $50 \mathrm{MW}$ & $11 / 132 \mathrm{kV}$ \\
\hline
\end{tabular}


Arsalan Muhammad Soomar, Abdul Hakeem Shaikh and Muhammad Hanif Lashari

Table 3 Summary of 220kV transmission line Capacity

\begin{tabular}{|c|c|c|c|c|}
\hline \multirow{2}{*}{ Name of T/line } & Control Breaker & $\begin{array}{c}\text { Length of line } \\
(\boldsymbol{K M})\end{array}$ & \multicolumn{2}{|c|}{ Conductor } \\
\cline { 4 - 5 } & (D3Q1) & 83.98 & $\begin{array}{c}\text { Greely } \\
\text { ACCR }\end{array}$ & 1582 \\
\hline TMK Road CKT-I & (D4Q & $\begin{array}{c}\text { Greely } \\
\text { ACCR }\end{array}$ & 1582 \\
\hline TMK Road CKT-II & 83.98 & \multicolumn{2}{|c|}{} \\
\hline
\end{tabular}

Table 4 Summary of $132 \mathrm{kV}$ transmission line Capacity

\begin{tabular}{|l|c|c|c|c|}
\hline \multirow{2}{*}{ Name of T/line } & \multirow{2}{*}{$\begin{array}{c}\text { Control } \\
\text { Breaker }\end{array}$} & \multirow{2}{*}{$\begin{array}{c}\text { Length of line } \\
(\boldsymbol{K M})\end{array}$} & \multicolumn{2}{|c|}{ Conductor } \\
\cline { 4 - 5 } & Type & $\begin{array}{c}\text { Capacity } \\
\text { (Amps) }\end{array}$ \\
\hline TMK City CKT-I & (E16Q1) & 75 & $\begin{array}{c}\text { Greely } \\
\text { ACCR }\end{array}$ & 791 \\
\hline TMK City CKT-II & (E17Q1) & 75 & $\begin{array}{c}\text { Greely } \\
\text { ACCR }\end{array}$ & 791 \\
\hline
\end{tabular}

Table 5 Summary of 132/220kV Capacity.

\begin{tabular}{|l|c|c|c|c|}
\hline \multirow{2}{*}{ Name of T/line } & \multirow{2}{*}{ Bus Bar } & \multirow{2}{*}{ CT Ratio } & \multicolumn{2}{|c|}{ Conductor } \\
\cline { 4 - 5 } & & & $\begin{array}{c}\text { Capacity } \\
\text { (Amps) }\end{array}$ \\
\hline 220 KV Bus Bar-I & 2 Bundled flexible & $2400 / 1$ & Coreopsis & 2416 \\
\hline 220 KV Bus Bar-II & 2 Bundled flexible & $2400 / 1$ & Coreopsis & 2416 \\
\hline 132 KV Bus Bar-I & Rigid & $1600 / 1$ & Coreopsis & 4000 \\
\hline 132 KV Bus Bar-II & Rigid & $1600 / 1$ & Coreopsis & 4000 \\
\hline
\end{tabular}

Table 6 Summary of Transformers capacity.

\begin{tabular}{|l|c|c|c|c|}
\hline \multirow{2}{*}{ Transformer } & \multirow{2}{*}{ Make } & \multirow{2}{*}{ Ratting } & \multicolumn{2}{c|}{ Capacity Amps } \\
\cline { 4 - 5 } Auto T/F T-1 & XIAN XD China & $\begin{array}{c}250 \mathrm{MVA}, \\
220 / 132 \mathrm{kV}\end{array}$ & 656.099 & 1093.498 \\
\hline Auto T/F T-2 & XIAN XD China & $\begin{array}{c}250 \mathrm{MVA}, \\
220 / 132 \mathrm{kV}\end{array}$ & 656.099 & 1093.498 \\
\hline Auto T/F T-3 & XIAN XD China & $\begin{array}{c}250 \mathrm{MVA}, \\
220 / 132 \mathrm{kV}\end{array}$ & 656.099 & 1093.498 \\
\hline Auto T/F T-4* & XIAN XD China & $\begin{array}{c}250 \mathrm{MVA}, \\
220 / 132 \mathrm{kV}\end{array}$ & 656.099 & 1093.498 \\
\hline Power T/F T-t & PEL & $\begin{array}{c}10 / 13 \mathrm{MVA}, \\
132 / 11 \mathrm{kV}\end{array}$ & 56.9 & 652.7 \\
\hline
\end{tabular}

*future addition

\section{RESULTS AND DISCUSSION}

\subsection{Wind Farm Running at Maximum Capacity}

In this case WTG are running at maximum installed capacity and power injected to $132 \mathrm{kV}$ bus with 2 incoming grid circuits of TMK and power is taken from $220 \mathrm{KV}$ two $\mathrm{kV}$ circuits as shown in Fig.1, Results of various equipment's and buses are shown in (Table VII-IX) respectively. 


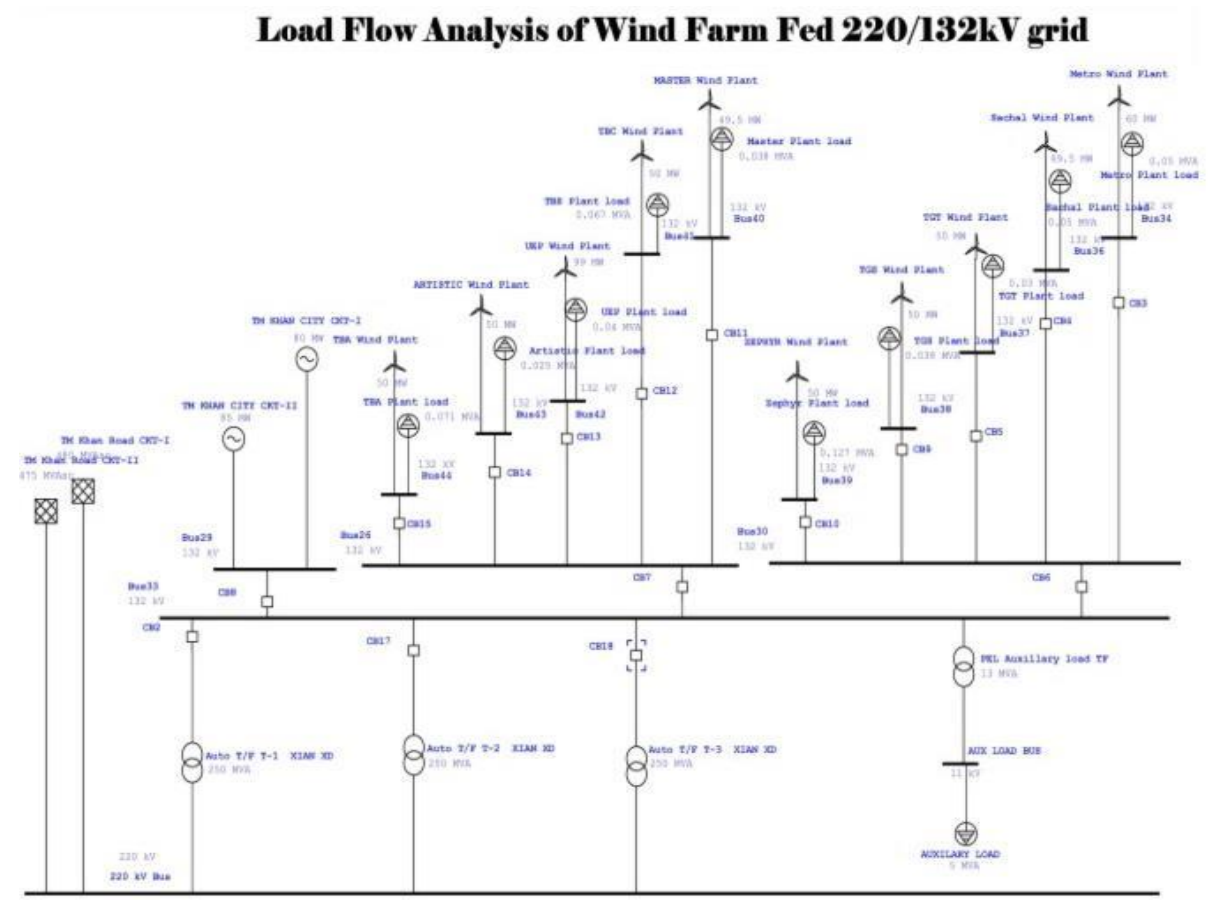

Figure 1. One line diagram of System under study in ETAP

Table 7 Summary of WTG Generation and Loading.

\begin{tabular}{|l|c|c|c|c|}
\hline \multicolumn{1}{|c|}{ Name of WTG } & Rating/Limit & MW & Amp & \% Generation \\
\hline ARTISTIC & $50 \mathrm{MW}$ & 50 & 218.7 & 100 \\
\hline MASTER & $49.5 \mathrm{MW}$ & 49.5 & 216.5 & 100 \\
\hline Metro & $60 \mathrm{MW}$ & 60 & 262.4 & 100 \\
\hline Sachal & $49.5 \mathrm{MW}$ & 49.5 & 216.5 & 100 \\
\hline TBA & $50 \mathrm{MW}$ & 50 & 218.7 & 100 \\
\hline TBC & $50 \mathrm{MW}$ & 50 & 218.7 & 100 \\
\hline TGS & $50 \mathrm{MW}$ & 50 & 218.7 & 100 \\
\hline TGT & $50 \mathrm{MW}$ & 50 & 218.7 & 100 \\
\hline UEP & $99 \mathrm{MW}$ & 99 & 433 & 100 \\
\hline ZEPHYR & $50 \mathrm{MW}$ & 50 & 218.7 & 100 \\
\hline
\end{tabular}

100

75

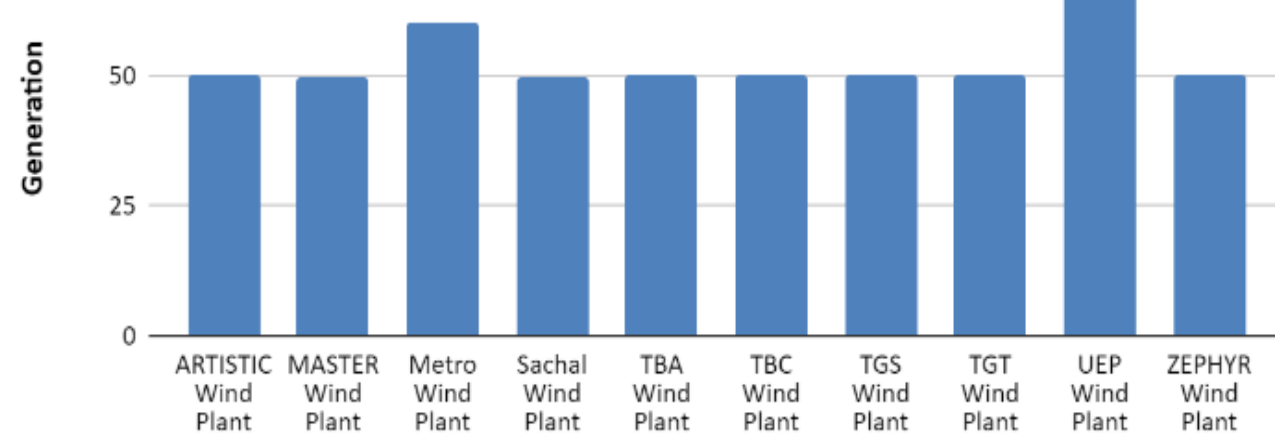

Figure 2. Generation at Maximum capacity 
Amp Loading vs. Name of WInd Turbine Generator

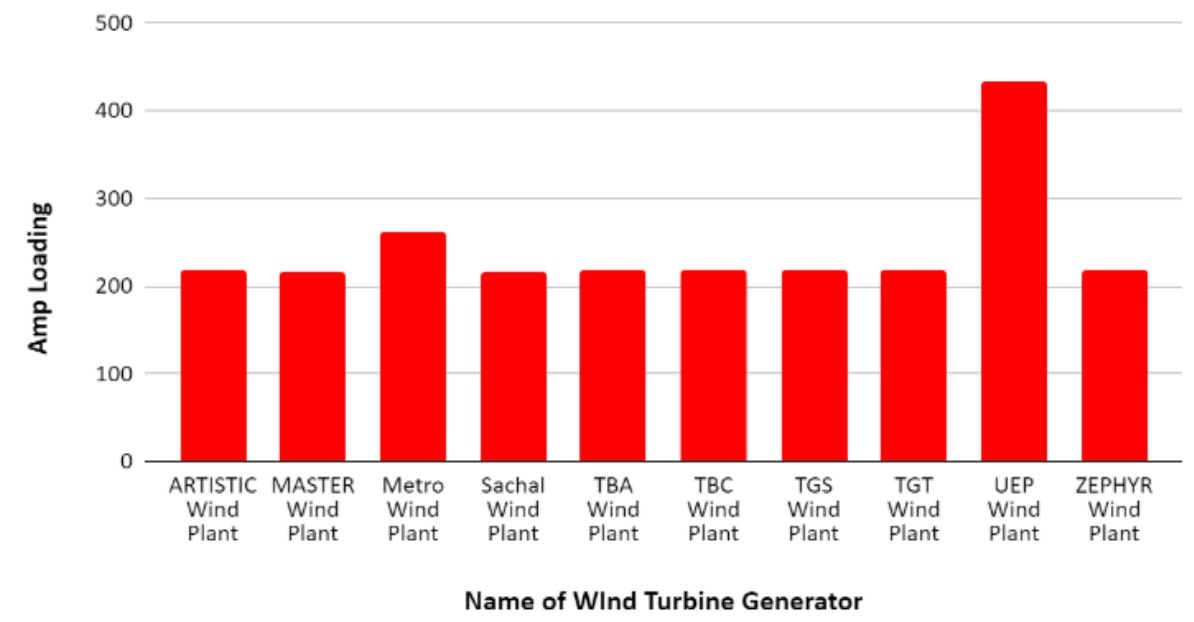

Figure 3. Ampere loading at Maximum capacity

Table 8 Power flow from 132/220kV transformer

\begin{tabular}{|l|c|c|c|c|}
\hline \multicolumn{1}{|c|}{ Transformers } & Rating & Allowable & kW Flow & kVAR Flow \\
\hline Auto T/F T-1 XIAN XD & $132 / 220 \mathrm{kV}$ & $250000 \mathrm{kVA}$ & 215473 & -16466.1 \\
\hline Auto T/F T-2 XIAN XD & $132 / 220 \mathrm{kV}$ & $250000 \mathrm{kVA}$ & 215473 & -16466.1 \\
\hline Auto T/F T-3 XIAN XD & $132 / 220 \mathrm{kV}$ & $250000 \mathrm{kVA}$ & 215473 & -16466.1 \\
\hline Auxiliary load TF & $132 / 11 \mathrm{kV}$ & $13000 \mathrm{kVA}$ & 25.5 & 15.81 \\
\hline
\end{tabular}

Table 9 Power flow from 132/220kV transmission line

\begin{tabular}{|l|c|c|c|c|c|}
\hline $\begin{array}{c}\text { Name of } \\
\text { bus }\end{array}$ & Rated kV & Type & Rating & MW & Amp \\
\hline $\begin{array}{l}\text { TMK CITY } \\
\text { CKT-I }\end{array}$ & 132 & Power Grid & $30 \mathrm{MW}$ & 30 & 132.6 \\
\hline $\begin{array}{l}\text { TMK CITY } \\
\text { CKT-II }\end{array}$ & 132 & Power Grid & $60 \mathrm{MW}$ & 60 & 272 \\
\hline $\begin{array}{l}\text { TMK Road } \\
\text { CKT-I }\end{array}$ & 220 & $\begin{array}{c}\text { Distribution } \\
\text { grid }\end{array}$ & 380 MVA & -323.2 & 850.7 \\
\hline $\begin{array}{l}\text { TMK Road } \\
\text { CKT-II }\end{array}$ & 220 & $\begin{array}{c}\text { Distribution } \\
\text { grid }\end{array}$ & 375 MVA & -323.2 & 850.7 \\
\hline
\end{tabular}

\subsection{Wind Farms Running at Different Capacities (As Per Grid Data)}

In this case WTG are running at different generation ratings capacity which are collected form the grid data here we will compare four months data i.e. (March, April, May, June) to analyze the power flow injected to $132 \mathrm{kV}$ bus with 2 incoming grid circuits of TMK and power fed to $220 \mathrm{kV}$ two circuits as shown in Fig.1, Results of various buses, transformers and generator loading are shown in Table X-XVIII respectively. 
Table 10 Summary of WTG Generation and Loading.

\begin{tabular}{|c|c|c|c|c|c|}
\hline \multirow[b]{2}{*}{ Name of WTG } & \multirow[b]{2}{*}{ Rated Capacity } & \multicolumn{2}{|c|}{ March } & \multicolumn{2}{|c|}{ April } \\
\hline & & MW Loading & $\begin{array}{c}\text { Amp } \\
\text { Loading }\end{array}$ & MW Loading & $\begin{array}{c}\text { Amp } \\
\text { Loading }\end{array}$ \\
\hline ARTISTIC & $50 \mathrm{MW}$ & 14 & 61.23 & 23 & 100.6 \\
\hline MASTER & $49.5 \mathrm{MW}$ & 9 & 39.36 & 13 & 56.86 \\
\hline Metro & $60 \mathrm{MW}$ & 20 & 87.48 & 22 & 96.23 \\
\hline Sachal & $49.5 \mathrm{MW}$ & 22 & 96.23 & 24 & 105 \\
\hline TBA & $50 \mathrm{MW}$ & 20 & 87.48 & 30 & 131.2 \\
\hline TBC & $50 \mathrm{MW}$ & 20 & 87.48 & 29 & 126.8 \\
\hline TGS & $50 \mathrm{MW}$ & 23 & 100.6 & 31 & 135.6 \\
\hline TGT & $50 \mathrm{MW}$ & 24 & 105 & 26 & 113.7 \\
\hline UEP & $99 \mathrm{MW}$ & 26 & 113.7 & 40 & 175 \\
\hline ZEPHYR & $50 \mathrm{MW}$ & 26 & 113.7 & 47 & 205.6 \\
\hline \multirow[b]{2}{*}{ Name of WTG } & \multirow[b]{2}{*}{ Rated Capacity } & \multicolumn{2}{|c|}{ May } & \multicolumn{2}{|c|}{ June } \\
\hline & & MW Loading & $\begin{array}{c}\text { Amp } \\
\text { Loading }\end{array}$ & MW Loading & $\begin{array}{c}\text { Amp } \\
\text { Loading }\end{array}$ \\
\hline ARTISTIC & $50 \mathrm{MW}$ & 33 & 144.3 & 30 & 131.2 \\
\hline MASTER & $49.5 \mathrm{MW}$ & 22 & 96.23 & 22 & 96.23 \\
\hline Metro & $60 \mathrm{MW}$ & 38 & 166.2 & 32 & 140 \\
\hline Sachal & $49.5 \mathrm{MW}$ & 42 & 183.7 & 40 & 175 \\
\hline TBA & $50 \mathrm{MW}$ & 43 & 188.1 & 41 & 179.3 \\
\hline TBC & $50 \mathrm{MW}$ & 42 & 183.7 & 40 & 175 \\
\hline TGS & $50 \mathrm{MW}$ & 47 & 205.6 & 48 & 209.9 \\
\hline TGT & $50 \mathrm{MW}$ & 46 & 201.2 & 47 & 205.6 \\
\hline UEP & $99 \mathrm{MW}$ & 58 & 253.7 & 55 & 240.6 \\
\hline ZEPHYR & $50 \mathrm{MW}$ & 64 & 279.9 & 32 & 140 \\
\hline
\end{tabular}

Table 11 Power flow from 132/220kV transformer

\begin{tabular}{|l|c|c|c|c|c|}
\hline Transformer Name & Rated kV & Allowable & kW Flow & $\begin{array}{c}\text { kVAR } \\
\text { Flow }\end{array}$ & $\begin{array}{c}\text { Amp } \\
\text { Flow }\end{array}$ \\
\hline $\begin{array}{l}\text { Auto T/F T-1 XIAN } \\
\text { XD }\end{array}$ & $\begin{array}{c}132 / 220 \\
\mathrm{kV}\end{array}$ & $250000 \mathrm{kVA}$ & 104774 & 416.8 & 458.3 \\
\hline $\begin{array}{l}\text { Auto T/F T-2 XIAN } \\
\text { XD }\end{array}$ & $\begin{array}{c}132 / 220 \\
\mathrm{kV}\end{array}$ & $250000 \mathrm{kVA}$ & 104774 & 416.8 & 458.3 \\
\hline $\begin{array}{l}\text { Auto T/F T-3 XIAN } \\
\text { XD }\end{array}$ & $\begin{array}{c}132 / 220 \\
\mathrm{kV}\end{array}$ & $250000 \mathrm{kVA}$ & 104774 & 416.8 & 458.3 \\
\hline Auxiliary load TF & $132 / 11 \mathrm{kV}$ & $13000 \mathrm{kVA}$ & 4221.7 & 2807.8 & 22.18 \\
\hline
\end{tabular}

Table 12 Power flow from 132/220kV transmission line

\begin{tabular}{|c|c|c|c|c|c|}
\hline Name of bus & Rated kV & Type & Rating & MW & Amp \\
\hline TMK CITY CKT-I & 132 & Power Grid & $30 \mathrm{MW}$ & 77 & 337.3 \\
\hline TMK CITY CKT-II & 132 & Power Grid & $60 \mathrm{MW}$ & 38 & 166.2 \\
\hline TMK Road CKT-I & 220 & $\begin{array}{c}\text { Distribution } \\
\text { grid }\end{array}$ & $380 \mathrm{MVA}$ & -156.98 & 412.4 \\
\hline TMK Road CKT-II & 220 & $\begin{array}{c}\text { Distribution } \\
\text { grid }\end{array}$ & $375 \mathrm{MVA}$ & -156.98 & 412.4 \\
\hline
\end{tabular}


Arsalan Muhammad Soomar, Abdul Hakeem Shaikh and Muhammad Hanif Lashari

Table 13 Power flow from 132/220kV transformer

\begin{tabular}{|l|c|c|c|c|c|}
\hline Transformer Name & Rated kV & Allowable & kW Flow & $\begin{array}{c}\text { kVAR } \\
\text { Flow }\end{array}$ & $\begin{array}{c}\text { Amp } \\
\text { Flow }\end{array}$ \\
\hline $\begin{array}{l}\text { Auto T/F T-1 XIAN } \\
\text { XD }\end{array}$ & $\begin{array}{c}132 / 220 \\
\mathrm{kV}\end{array}$ & $250000 \mathrm{kVA}$ & 104774 & 416.8 & 458.3 \\
\hline $\begin{array}{l}\text { Auto T/F T-2 XIAN } \\
\text { XD }\end{array}$ & $\begin{array}{c}132 / 220 \\
\mathrm{kV}\end{array}$ & $250000 \mathrm{kVA}$ & 104774 & 416.8 & 458.3 \\
\hline $\begin{array}{l}\text { Auto T/F T-3 XIAN } \\
\text { XD }\end{array}$ & $\begin{array}{c}132 / 220 \\
\mathrm{kV}\end{array}$ & $250000 \mathrm{kVA}$ & 104774 & 416.8 & 458.3 \\
\hline Auxiliary load TF & $132 / 11 \mathrm{kV}$ & $13000 \mathrm{kVA}$ & 4221.7 & 2807.8 & 22.18 \\
\hline
\end{tabular}

Generation Comparison of Plants

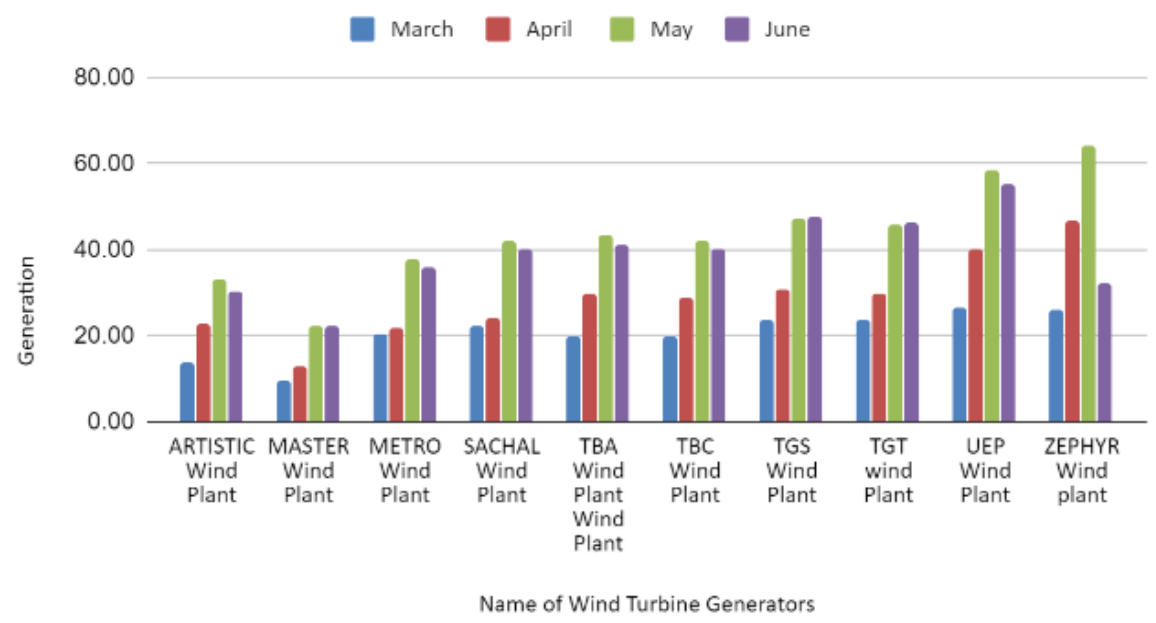

Figure 4 Generation at Different capacities

Amp Loading of the WInd Turbine generators \& buses

March $\square$ April $\square$ May $\square$ June

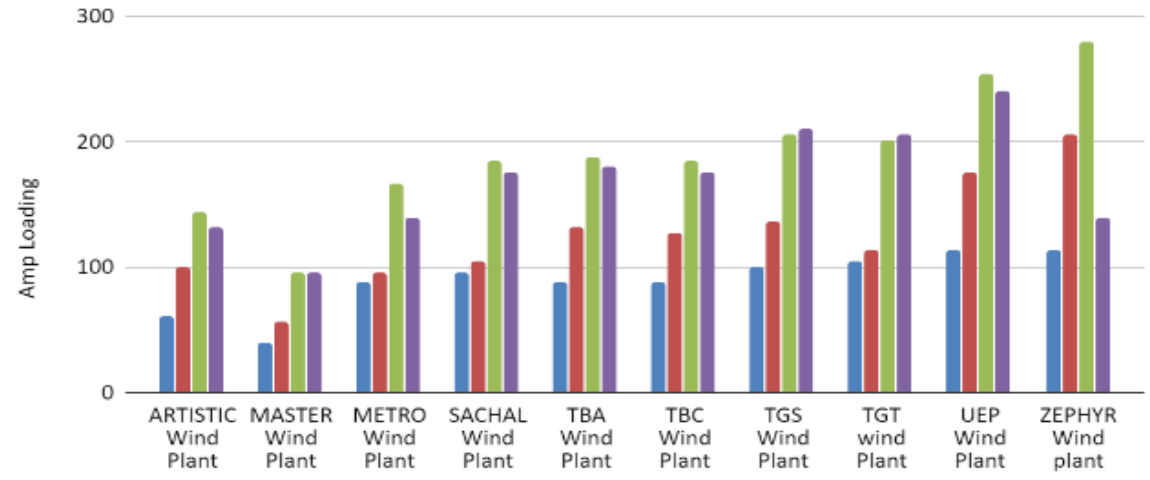

Name of Wind Turbine Generators

Figure 5 Ampere loading at Different capacities 
Table 14 Power flow of 132/220kV transmission line

\begin{tabular}{|l|c|c|c|c|c|}
\hline \multicolumn{1}{|c|}{ Name of bus } & $\begin{array}{c}\text { Rated } \\
\mathbf{k V}\end{array}$ & Type & Rating & MW & Amp \\
\hline TMK CITY CKT-I & 132 & Power Grid & $30 \mathrm{MW}$ & 77.1 & 343.7 \\
\hline $\begin{array}{l}\text { TMK CITY CKT- } \\
\text { II }\end{array}$ & 132 & Power Grid & $60 \mathrm{MW}$ & 77 & 336.8 \\
\hline TMK Road CKT-I & 220 & Distribution grid & $380 \mathrm{MVA}$ & -267.69 & 704.1 \\
\hline $\begin{array}{l}\text { TMK Road CKT- } \\
\text { II }\end{array}$ & 220 & Distribution grid & $375 \mathrm{MVA}$ & -267.69 & 704.1 \\
\hline
\end{tabular}

Table 15 Power flow from 132/220kV transformer

\begin{tabular}{|l|c|c|c|c|c|}
\hline \multicolumn{1}{|c|}{$\begin{array}{c}\text { Transformer } \\
\text { Name }\end{array}$} & Rated kV & Allowable & kW Flow & $\begin{array}{c}\text { kVAR } \\
\text { Flow }\end{array}$ & $\begin{array}{c}\text { Amp } \\
\text { Flow }\end{array}$ \\
\hline $\begin{array}{l}\text { Auto T/F T-1 XIAN } \\
\text { XD }\end{array}$ & $\begin{array}{c}132 / 220 \\
\mathrm{kV}\end{array}$ & $250000 \mathrm{kVA}$ & 191365 & -13455 & 503.4 \\
\hline $\begin{array}{l}\text { Auto T/F T-2 XIAN } \\
\text { XD }\end{array}$ & $\begin{array}{c}132 / 220 \\
\mathrm{kV}\end{array}$ & $250000 \mathrm{kVA}$ & 191365 & -13455 & 503.4 \\
\hline $\begin{array}{l}\text { Auto T/F T-3 XIAN } \\
\text { XD }\end{array}$ & $\begin{array}{c}132 / 220 \\
\mathrm{kV}\end{array}$ & $250000 \mathrm{kVA}$ & 191365 & -13455 & 503.4 \\
\hline Auxiliary load TF & $132 / 11 \mathrm{kV}$ & $13000 \mathrm{kVA}$ & 4221.7 & 2807.8 & 22.18 \\
\hline
\end{tabular}

Table 15 Power flow from 132/220kV transmission line

\begin{tabular}{|l|c|c|c|c|c|}
\hline \multicolumn{1}{|c|}{ Name of bus } & $\begin{array}{c}\text { Rated } \\
\mathbf{k V}\end{array}$ & Type & Rating & MW & Amp \\
\hline TMK CITY CKT-I & 132 & Power Grid & $30 \mathrm{MW}$ & 73 & 328.8 \\
\hline $\begin{array}{l}\text { TMK CITY CKT- } \\
\text { II }\end{array}$ & 132 & Power Grid & $60 \mathrm{MW}$ & 72 & 314.9 \\
\hline TMK Road CKT-I & 220 & $\begin{array}{c}\text { Distribution } \\
\text { grid }\end{array}$ & 380 MVA & -287.048 & 755.2 \\
\hline TMK Road CKT-II & 220 & $\begin{array}{c}\text { Distribution } \\
\text { grid }\end{array}$ & 375 MVA & -287.048 & 755.2 \\
\hline
\end{tabular}

Table 16 Power flow from 132/220kV transmission line

\begin{tabular}{|l|c|c|c|c|c|}
\hline \multicolumn{1}{|c|}{$\begin{array}{c}\text { Transformer } \\
\text { Name }\end{array}$} & Rated kV & Allowable & kW Flow & kVAR Flow & $\begin{array}{c}\text { Amp } \\
\text { Flow }\end{array}$ \\
\hline $\begin{array}{l}\text { Auto T/F T-1 XIAN } \\
\text { XD }\end{array}$ & $\begin{array}{c}132 / 220 \\
\mathrm{kV}\end{array}$ & $250000 \mathrm{kVA}$ & 178452 & -11964.7 & 469.4 \\
\hline $\begin{array}{l}\text { Auto T/F T-2 XIAN } \\
\text { XD }\end{array}$ & $\begin{array}{c}132 / 220 \\
\mathrm{kV}\end{array}$ & $250000 \mathrm{kVA}$ & 178452 & -11964.7 & 469.4 \\
\hline $\begin{array}{l}\text { Auto T/F T-3 XIAN } \\
\text { XD }\end{array}$ & $\begin{array}{c}132 / 220 \\
\mathrm{kV}\end{array}$ & $250000 \mathrm{kVA}$ & 178452 & -11964.7 & 469.4 \\
\hline Auxiliary load TF & $132 / 11 \mathrm{kV}$ & $13000 \mathrm{kVA}$ & 4221.7 & 2807.8 & 22.18 \\
\hline
\end{tabular}


Table 17 Power flow from 132/220kV transmission line

\begin{tabular}{|l|c|c|c|c|c|}
\hline \multicolumn{1}{|c|}{ Name of bus } & $\begin{array}{c}\text { Rated } \\
\mathbf{k V}\end{array}$ & Type & Rating & MW & Amp \\
\hline TMK CITY CKT-I & 132 & Power Grid & $30 \mathrm{MW}$ & 77.1 & 343.7 \\
\hline $\begin{array}{l}\text { TMK CITY CKT- } \\
\text { II }\end{array}$ & 132 & Power Grid & $60 \mathrm{MW}$ & 77 & 336.8 \\
\hline TMK Road CKT-I & 220 & Distribution grid & $380 \mathrm{MVA}$ & -267.68 & 704.1 \\
\hline TMK Road CKT-II & 220 & Distribution grid & $375 \mathrm{MVA}$ & -267.68 & 704.1 \\
\hline
\end{tabular}

\subsection{Wind Farms Response After Adding One More Autotransformer in Grid}

In this case WTG are running at optimum ratings capacity and the new transformer of 250MVA is added to the simulation which is going to be installed in future at the present grid to increase its power capacity to accommodate more power, which will allow to take more power from the $132 \mathrm{kV}$ TMK city grid circuits and wind power stations, so that it can transfer more power to the load side which is $220 \mathrm{kV}$ two circuits connecting TMK Road Grid as shown in Fig.3-4, Results of various generators, buses and transformer loading are shown in Table XIX-XXII respectively to examine the load flow so that all bus, transformer are well under limits according to the software calculation.

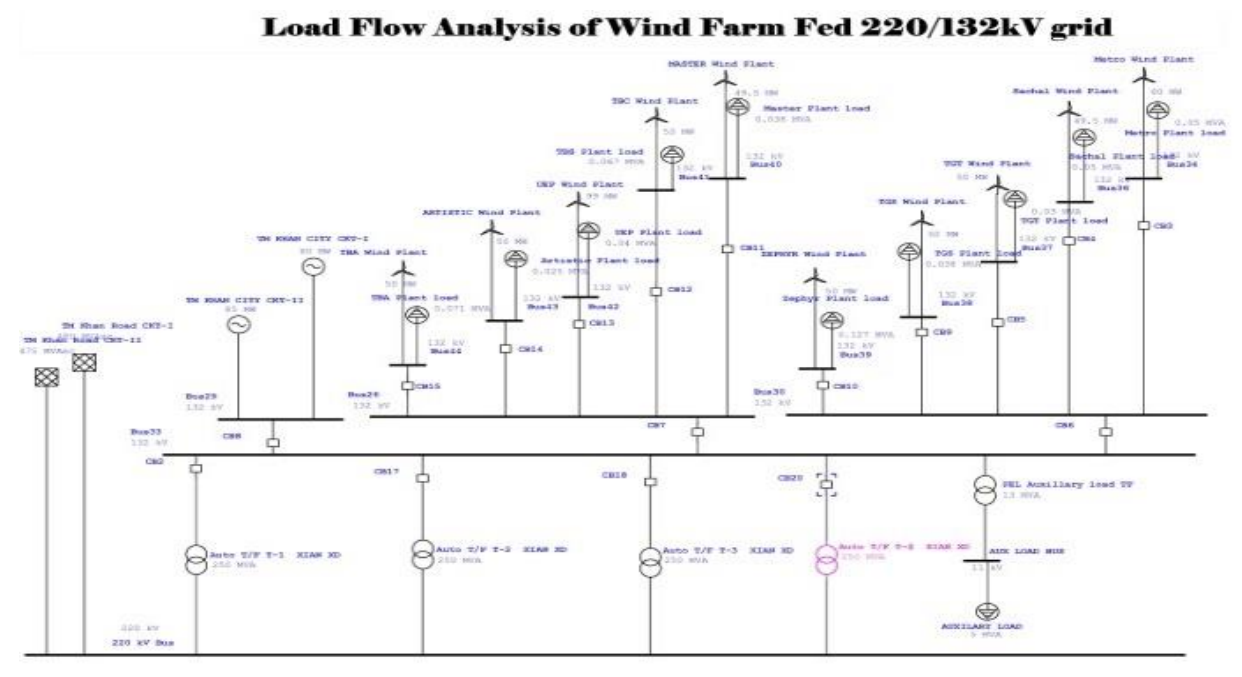

Figure 3 One-line diagram after expansion of system in ETAP

Table 18 Summary of generation and loading

\begin{tabular}{|l|c|c|c|c|}
\hline Name of WTG & Rated Capacity & Opt Capacity & Amp Loading & \% Generation \\
\hline ARTISTIC & $50 \mathrm{MW}$ & $50 \mathrm{MW}$ & 218.7 & 100 \\
\hline MASTER & $49.5 \mathrm{MW}$ & $49.5 \mathrm{MW}$ & 216.5 & 100 \\
\hline Metro & $60 \mathrm{MW}$ & $60 \mathrm{MW}$ & 262.4 & 100 \\
\hline Sachal & $49.5 \mathrm{MW}$ & $49.5 \mathrm{MW}$ & 216.5 & 100 \\
\hline TBA & $50 \mathrm{MW}$ & $50 \mathrm{MW}$ & 218.7 & 100 \\
\hline TBC & $50 \mathrm{MW}$ & $50 \mathrm{MW}$ & 218.7 & 100 \\
\hline TGS & $50 \mathrm{MW}$ & $50 \mathrm{MW}$ & 218.7 & 100 \\
\hline TGT & $50 \mathrm{MW}$ & $50 \mathrm{MW}$ & 218.7 & 100 \\
\hline UEP & $99 \mathrm{MW}$ & $99 \mathrm{MW}$ & 433 & 100 \\
\hline ZEPHYR & $50 \mathrm{MW}$ & $50 \mathrm{MW}$ & 218.7 & 100 \\
\hline
\end{tabular}


Table 19 Summary of generation and loading

\begin{tabular}{|l|c|c|c|c|}
\hline \multicolumn{1}{|c|}{ Transformers } & Rating & kW Flow & kVAR Flow & Amp Flow \\
\hline Auto T/F T-1 XIAN XD & $132 / 220 \mathrm{kV}$ & 160543 & -10037.9 & 422.1 \\
\hline Auto T/F T-2 XIAN XD & $132 / 220 \mathrm{kV}$ & 160543 & -10037.9 & 422.1 \\
\hline Auto T/F T-3 XIAN XD & $132 / 220 \mathrm{kV}$ & 160543 & -10037.9 & 422.1 \\
\hline Auto T/F T-4 XIAN XD & $132 / 220 \mathrm{kV}$ & 160543 & -10037.9 & 422.1 \\
\hline PEL Auxiliary load TF & $132 / 11 \mathrm{kV}$ & 4221.7 & 2807.8 & 22.18 \\
\hline
\end{tabular}

Table 20 Summary of generation and loading

\begin{tabular}{|l|l|c|c|c|}
\hline \multicolumn{1}{|c|}{ Bus Name } & \multicolumn{1}{c|}{ Type } & Rating & MW Loading & Amp \\
\hline TMK CITY CKT-I & Power Grid & $30 \mathrm{MW}$ & 30 & 131.9 \\
\hline TMK CITY CKT-II & Power Grid & $60 \mathrm{MW}$ & 60 & 267.3 \\
\hline TMK Road CKT-I & Distribution Grid & $380 \mathrm{MVA}$ & -321.086 & 844.3 \\
\hline TMK Road CKT-II & Distribution Grid & $375 \mathrm{MVA}$ & -321.086 & 844.3 \\
\hline
\end{tabular}

\subsection{Response of Grid on Losing Generator}

In this case WTG are running at nominal ratings capacity then one of generator is lose after that we will see the results of various equipment's and buses as shown in Table. XXII-XXIV) respectively to examine the load flow on $132 \mathrm{kV}$ TMK and load side which is $220 \mathrm{kV}$ two circuits connecting TMK Road Grid behavior as shown in Fig.7.

Table 21 Summary of WTG Generation and Loading

\begin{tabular}{|l|c|c|c|c|}
\hline Name of WTG & Rated Capacity & $\begin{array}{c}\text { Opt. } \\
\text { MW }\end{array}$ & Amp Loading & \% Generation \\
\hline ARTISTIC & $50 \mathrm{MW}$ & 25 & 109.3 & 49 \\
\hline MASTER & $49.5 \mathrm{MW}$ & 18 & 78.73 & 36 \\
\hline Metro & $60 \mathrm{MW}$ & 32 & 140 & 53 \\
\hline Sachal & $49.5 \mathrm{MW}$ & 35 & 153.1 & 71 \\
\hline TBA & $50 \mathrm{MW}$ & 33 & 144.3 & 66 \\
\hline TBC & $50 \mathrm{MW}$ & 33 & 144.3 & 65 \\
\hline TGS & $50 \mathrm{MW}$ & 38 & 166.2 & 78 \\
\hline TGT & $50 \mathrm{MW}$ & 37 & 161.8 & 76 \\
\hline UEP & $99 \mathrm{MW}$ & 45 & 196.8 & 45 \\
\hline ZEPHYR & $50 \mathrm{MW}$ & 0 & - & - \\
\hline
\end{tabular}

Table 22 Summary of WTG Generation and Loading

\begin{tabular}{|l|c|c|c|c|}
\hline \multicolumn{1}{|c|}{ Transformers } & Rating & kW Flow & kVAR Flow & Amp Flow \\
\hline Auto T/F T-1 XIAN XD & $132 / 220 \mathrm{kV}$ & 151850 & -9161.5 & 399.2 \\
\hline Auto T/F T-2 XIAN XD & $132 / 220 \mathrm{kV}$ & 151850 & -9161.5 & 399.2 \\
\hline Auto T/F T-3 XIAN XD & $132 / 220 \mathrm{kV}$ & 151850 & -9161.5 & 399.2 \\
\hline PEL Auxiliary load TF & $132 / 11 \mathrm{kV}$ & 4221.7 & 2807.8 & 22.18 \\
\hline
\end{tabular}


MW loading vs. Name of WTG

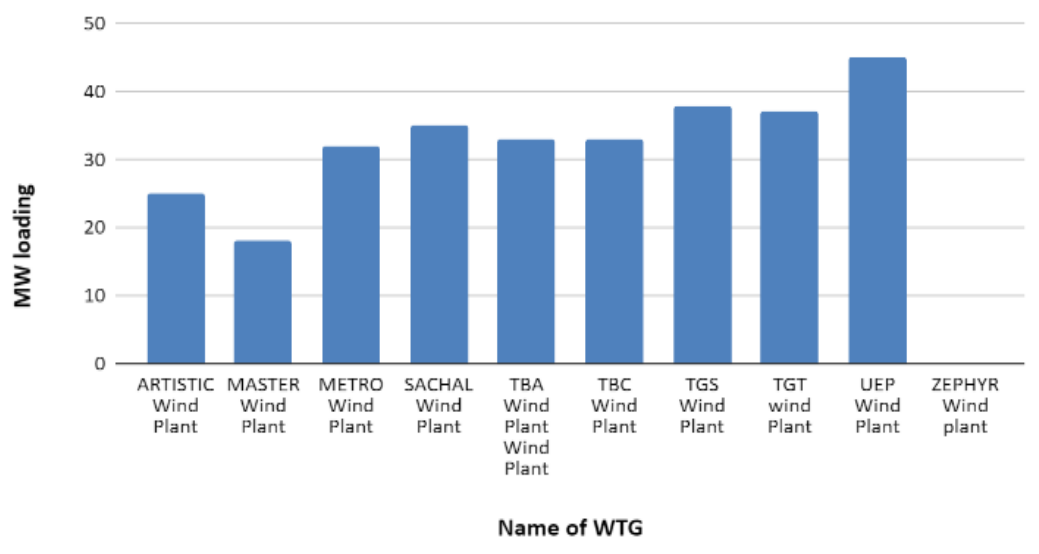

Figure 7 Generation at losing one Generator

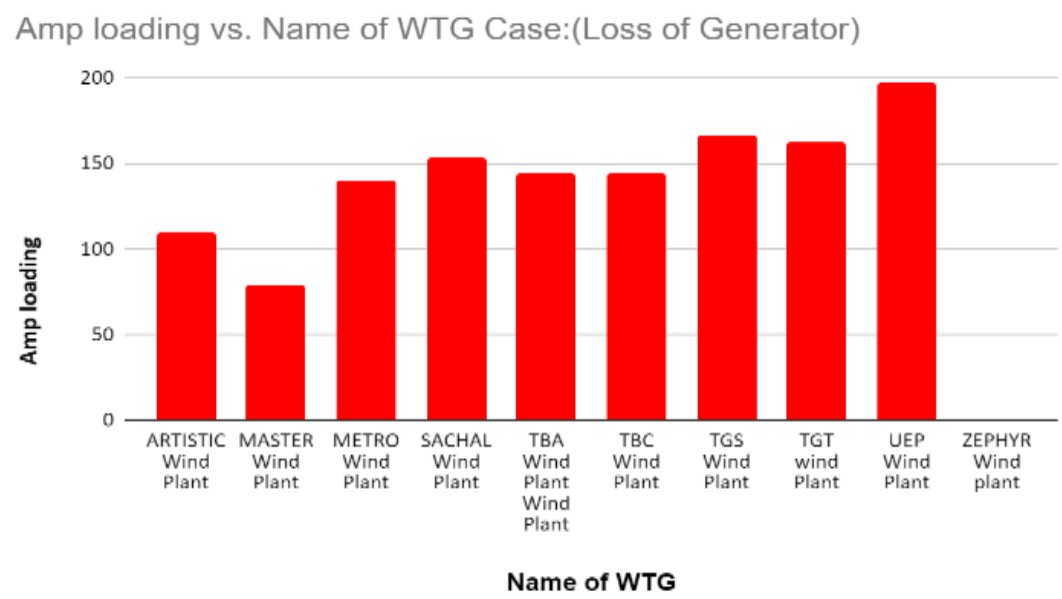

Figure 8 Ampere loading at losing one Generator

Table 23 Power flow from 132/220kV transmission line

\begin{tabular}{|l|l|c|c|c|}
\hline \multicolumn{1}{|c|}{ Name of T/L } & \multicolumn{1}{c|}{ Type } & Rating & Opt limit & Amp \\
\hline TMK CITY CKT-I & Power Grid & $200 \mathrm{MW}$ & 80 & 349.9 \\
\hline TMK CITY CKT-II & Power Grid & $200 \mathrm{MW}$ & 85 & 374.5 \\
\hline TMK Road CKT-I & $\begin{array}{l}\text { Distribution } \\
\text { Grid }\end{array}$ & $500 \mathrm{MVA}$ & -227.78 & 598.8 \\
\hline TMK Road CKT-II & $\begin{array}{l}\text { Distribution } \\
\text { Grid }\end{array}$ & $500 \mathrm{MVA}$ & -227.78 & 598.8 \\
\hline
\end{tabular}

\section{CONCLUSION}

Load flow analysis for the future expansion of the grid is being successful carried out in this paper we observed, Simulation for the various cases such as optimum load capacity with maximum generation allow us to see the behavior of buses at maximum capacity without any fault system will remain intact., in this second case, the loss of generator we also successfully analyze the loading of other generators are well under capacity and for the future load addition and expansion of the grid adding of new circuits for extension its capacity with the inclusion of one transformer into the grid to increase its capacity from 750 to $1250 \mathrm{MW}$ and we have 
analyzed from the simulation that all buses are performing well under the specified limits and capacity to continue safe operation to flow maximum power without any hindrance and loading to its maximum possible condition,

On basis of these cases we can do changes to maintain our systems reliability and safe operation, and future expansion so that more power deliver to the user end, and cheapest power from renewable sources can be injected from this grid. simulation results confirms the effectiveness of the proposed work.

\section{ACKNOWLEDGMENT}

Authors are thankful to Mehran University of Engineering and Technology for providing necessary resources and guidance during the Post graduation studies.

\section{REFERENCES}

[1] Albadi, M. (2019) "Power Flow Analysis", Intechopen.83374

[2] Gomez, A.V (2019), "Load Flow Analysis of 138/69kV Substation Using Electrical Transient \& Analysis Program (ETAP)," Electrical Engineering Undergraduate Honors Theses. 67.

[3] Kamble, S.C: Prof. Rajni Rewatkar, (2017) "Load-Flow Analysis of Distribution Systems Using ETAP”, International Journal of Advanced Engineering, Management and Science, Special Issue-2.

[4] Kapahi, R. (2013) "Load Flow Analysis of $132 \mathrm{kV}$ Substation Using ETAP" Software, International Journal of Scientific \& Engineering Research Volume 4, Issue 2

[5] Mumtaz, M.: Hussain Sarwar Khan, Muhammad Aamir, Muhammad Ali, Ali Rehman, (2018) "Load Flow Analysis of Cigre Benchmark Model Using ETAP", International Conference on Renewable, Applied and New Energy Technologies Icranet, 19-22

[6] Natkar, K, Naveen Kumar, (2015)" Design Analysis of 220/132 kV Substation Using ETAP”, International Research Journal of Engineering and Technology (Irjet), Volume: 02 Issue: 03.

[7] Patel, Y.: Dixit Tandel, Dharti Katti, (2014) "Simulation and Analysis of 220kV Substation", International Journal of Advanced Research in Electrical, Electronics and Instrumentation Engineering, Vol. 3, Issue.

[8] Rehman, Z: Waqas Hussain, Rizwan Ullah and Zaki-Ud-Din, (2017) "Load Flow Analysis of 132/11kV Substation Using ETAP: A Case Study" Sarhad University International Journal of Basic and Applied Sciences, Vol 5.

[9] Salgar, A.S., Prof. Ch. Mallareddy, (2015)" Load Flow Analysis for A 220kV Line - Case Study", International Journal of Innovations in Engineering Research and Technology [Ijiert] Issn: 2394-3696 Volume 2, Issue 5.

[10] Shinde, K.S: Atul M. Shewale, "Load Flow Analysis of 220 kV MSETCL Substation by Using ETAP”, Elsevier Science \& Technology, Issn No. 978-93-5107-223-2. 